\title{
Estudio Biométrico de la Distribución de los Ramos Motores del Nervio Fibular Superficial en el Compartimiento Lateral de la Pierna en Individuos Brasileiros: Implicancias Clínicas y Variaciones
}

\author{
Biometric study of the distribution of the motor branches of the superficial fibular nerve in \\ the lateral compartment of the leg in Brazilian individuals: clinical implications and variations
}

Pelozo Jr, O. ${ }^{\text {; }}$ Garbelotti Jr, S.A. ${ }^{2}$; Itezeroti, A.M. ${ }^{1}$; Cricenti, S.V. ${ }^{2}$; de Angelis, M.A. ${ }^{2}$ \& Olave , E. ${ }^{3}$

PELOZO JR, O.; GARBElotTi JR, S. A.; ITEZEROTI, A. M.; CRICENTI, S. V. ; DE ANGELIS, M. A. \& OLAVE, E. Estudio biométrico de la distribución de los ramos motores del nervio fibular superficial en el compartimiento lateral de la pierna en individuos Brasileiros: implicancias clínicas y variaciones. Int. J. Morphol., 36(4):1457-1452, 2018.

RESUMEN: El propósito del presente estudio fue conocer la distribución de los ramos motores del nervio fibular superficial (NFS) y de sus respectivas penetraciones en los músculos fibulares en relación al ápice de la cabeza de la fíbula, dividiendo el compartimiento lateral de la pierna en tres regiones a fin de hacer posible una visión más segura de sus correlaciones clínicas y quirúrgicas. A través de disección, se estudiaron 60 piernas pareadas de 30 cadáveres adultos, de ambos sexos, Brasileños, con edad promedio de 44,9 años, siendo 8 de sexo femenino y 22 del masculino. Después de la disección se registraron las distancias de los puntos de penetración de los ramos del NFS en los músculos fibular largo $(\mathrm{mFL})$ y corto $(\mathrm{mFC})$, localizándolos en los tercios proximal, medio o distal, según fuere el caso. Se observó que el mayor número de ramos penetraron en el mFL a nivel de la parte distal del tercio proximal de la pierna, mientras que en el mFC lo hicieron en las partes proximal y distal del tercio medio de la pierna. Los ramos motores para el mFL penetraban en el vientre muscular entre 48,06 y 141,56 mm, y los ramos para el mFC lo hicieron entre 163,34 y $209,67 \mathrm{~mm}$ del origen del nervio. No hubo diferencias estadísticamente significativas ni entre los lados derecho e izquierdo ni entre genéros. Independiente de las diferencias metodológicas entre los estudios disponibles, el detalle de la distribución nerviosa en este compartimiento, permitirá una mayor precisión en el momento de elegirse un área para colgajo de injerto autólogo y una menor chance de lesiones iatrogénicas durante cirugías de la región.

PALABRAS CLAVE: Anatomía; Nervio fibular superficial; Músculos fibulares; Variaciones anatómicas.

\section{INTRODUCCIÓN}

El nervio fibular superficial (NFS) es un ramo terminal de característica músculo-cutánea, responsable por la inervación de los músculos fibular largo $(\mathrm{mFL})$ y fibular corto (mFC). Durante su trayecto en el compartimiento lateral de la pierna, perfora el $\mathrm{mFL}$ y continua sobre el $\mathrm{mFC}$, descendiendo entre ellos y el músculo extensor largo de los dedos para tornarse superficial en el tercio distal de la pierna, pasando sobre el retináculo de los músculos extensores y dividiéndose en nervios cutáneos dorsales medial e intermedio, responsables por la inervación del dorso del pie (Büyükmumcu et al., 1999; Iannotti \& Parker, 2013; Ribak et al., 2016; Standring, 2016).

\footnotetext{
${ }^{1}$ Faculdade de Medicina Santa Marcelina, São Paulo, São Paulo, Brasil

${ }^{2}$ Universidade Federal de São Paulo, São Paulo, Brasil.

${ }^{3}$ Facultad de Medicina, Universidad de La Frontera, Temuco, Chile.
}

Los avances en la reconstrucción microquirúrgica han desempeñado papeles importantes en la mejoría de los resultados del tratamiento quirúrgico de las lesiones nerviosas. En este sentido, el injerto de nervio autólogo es necesario para completar una gran laguna nerviosa después de un trauma y los nervios cutáneos de los miembros superiores son comúnmente usados para este procedimiento. En los casos de injertos de nervios múltiples o muy largos es necesario utilizar otros nervios cutáneos tales como: nervios cutáneos surales o femorales además del NFS (Büyükmumcu et al.; Pacha et al., 2003; Agthong et al., 2008; RodriguezLorenzo et al., 2011). 
Así, el conocimiento detallado de la anatomía del NFS es importante para facilitar la selección y retirada del colgajo para injerto, además de entregar datos precisos para evitar lesión iatrogénica del nervio lo que invariablemente causaría incapacidad de eversión además de parestesia en la región lateral de la pierna y dorso del pie. Tanto Ducic et al. (2006), Barrett et al. (2006), Prakash et al. (2010) y Ribak et al. han alertado sobre la falta de atención del NFS en la literatura y han resaltado que esos datos buscan preparar mejor al cirujano en el manejo del NFS, en procedimientos de resección, artroscopías, bloqueo con anestésicos locales, neurolisis o fasciotomía para la transposición de colgajos en la región.

Nuestro objetivo fue estudiar detalladamente las variaciones de la distribución de los ramos del NFS, adoptando como referencia un mapeo regional de los orígenes y los respectivos puntos de penetración en los vientres de los $\mathrm{mFL}$ y $\mathrm{mFC}$, en relación al ápice de la cabeza de la fíbula, con el fin de posibilitar una visión más segura de sus correlaciones anátomo-clínicas y quirúrgicas.

\section{MATERIAL Y MÉTODO}

A través de disección, se estudiaron 60 piernas pareadas de 30 cadáveres formolizados, adultos, de ambos sexos, Brasileños, con edad promedio de 44,9 años, siendo 8 de sexo femenino y 22 del masculino, pertenecientes a la Disciplina de Anatomía Descriptiva y Topográfica de la Universidad Federal de Sã Paulo (UNIFESP-EPM), Brasil.

Se realizó una incisión en la superficie lateral de la pierna, desde la cabeza de la fíbula hasta el máleolo lateral, removiendo la piel alrededor de la pierna, dejando expuestos los músculos fibulares completamente. Los cadáveres fueron disecados siempre en una posición intermedia, entre decúbito dorsal y lateral, de manera que el compartimiento lateral de la pierna estuviera en una posición adecuada para la disección y registro de las medidas de los puntos de penetración de los ramos motores en los respectivos músculos.

Después de la disección se registraron las distancias de los puntos de penetración (P) de los ramos del NFS en los músculos mFL y $\mathrm{mFC}$, localizándolos en los tercios proximal, medio o distal, según fuere el caso. En cada tercio se consideró una parte proximal y otra distal, para tener resultados más detallados.

El nervio fibular común (NFC) fue expuesto desde la fosa poplítea y en su trayecto por la parte lateral de la rodilla hasta su penetración en la parte proximal del mFL. Este músculo fue cuidadosamente desinsertado del cóndilo lateral de la tibia, de la cabeza y del tercio proximal de la fíbula, con el propósito de exponer la bifurcación del nervio fibular común en su contorno por el cuello de la fíbula.

A partir del punto de bifurcación del NFC en nervio fibular profundo (NFP) y NFS, este último fue disecado en toda su extensión así como sus ramos de distribución para los músculos mFL y mFC. Luego, con un paquímetro digital de precisión 0,05 mm, marca Mitutoyo, se registraron las distancias entre el ápice de la cabeza de la fíbula y el punto en que esos ramos penetraron en el vientre muscular (Reebye, 2004). Las medidas registradas se correlacionaron con el tamaño de la fíbula, medido por una línea de referencia entre el ápice de su cabeza y la parte más distal del maléolo lateral. El valor fue expresado como la distancia porcentual a lo largo de la línea de referencia, utilizando el ápice de la cabeza como punto de partida, procedimiento similar al descrito por Lee et al. (2011).

Todos los datos fueron analizados usando SPSS (versión 20.0; SPSS Inc., Chicago, IL). Para analizar posibles diferencias entre los lados derecho e izquierdo, se usó el test no paramétrico para dos muestras relacionadas de Wilcoxon. Para la comparación entre sexos, se utilizó el test no paramétrico para dos muestras independientes de MannWhitney. Se consideró un intervalo de confianza del $95 \%$.

\section{RESULTADOS}

El número de puntos de penetración en el vientre muscular por parte del nervio es representado por $\mathrm{P}$, los promedios de las distancias fueron calculadas por cada punto en orden de aparción, de proximal a distal.

En las Tablas I y II se muestran los promedios generales de distancia de la distribución de los puntos de penetración en los músculos mFL y mFC, a partir del ápice de la cabeza de la fíbula y la normalización de los datos en porcentaje de la longitud de la fíbula. Los datos son presentados en promedios generales de lados derecho e izquierdo y de sexo masculino y femenino, no encontrándose diferencias significativas cuando se compararon los resultados entre lados o entre géneros $(p>0,05)$.

Por otra parte, los puntos de penetración P4 y P5 de la parte proximal del tercio proximal; P5, P6 y P7 de la parte distal del tercio superior y todos los puntos de penetración de la parte proximal del tercio medio del mFL, además de los puntos $\mathrm{P} 3$ del tercio medio proximal y P2 del tercio medio distal en el $\mathrm{mFC}$ no presentaron una cantidad suficiente de datos para análisis de diferencia estadística. 
No se observaron puntos de penetración de ramos motores en el tercio distal de ambos músculos.

Músculo Fibular Largo. En total, se observaron 426 puntos de penetración de ramos motores en este músculo, siendo $210(49,3 \%)$ en piernas derechas y $216(50,7 \%)$ en izquierdas. Del total, se encontraron 180 puntos $(42,3 \%)$ en la parte proximal del tercio proximal, $214(50,2 \%)$ en la parte distal del tercio proximal y $32(7,5 \%)$ en la parte proximal del tercio medio.

En la Tabla I se presentan el número de puntos de penetración de los ramos nerviosos en el mFL en los tercios

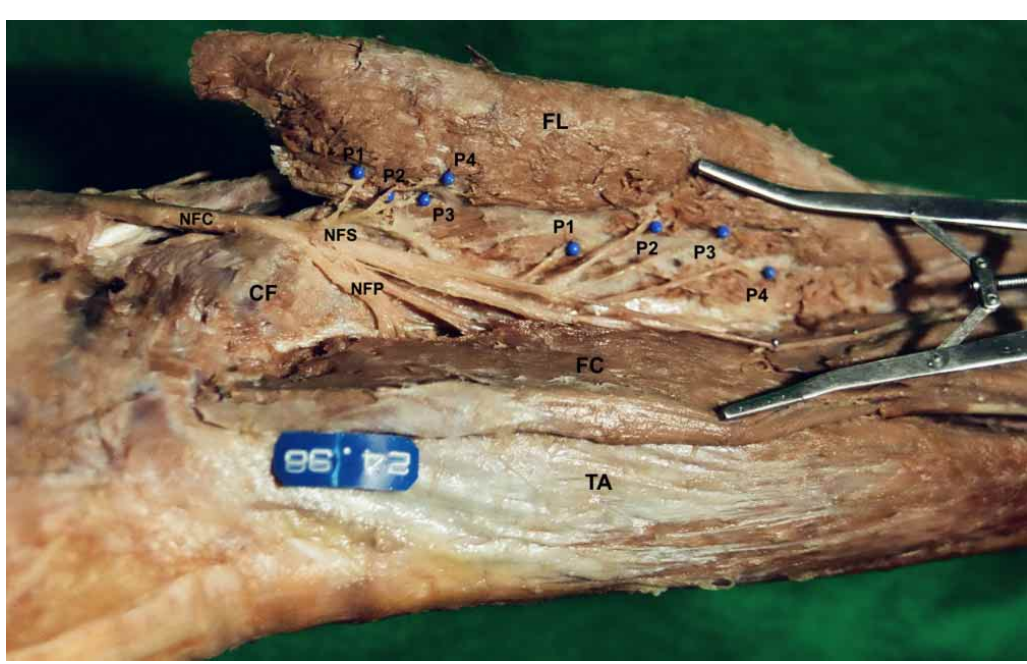

Fig. 1. Compartimiento lateral de una pierna izquierda, demostrando la ramificación del NFS en cuatro ramos motores (marcadores azules; P1-P4) tanto en el tercio superior proximal así como en el tercio superior distal para el mFL. NFC: nervio fibular común; CF: cabeza de la fíbula; NFP: nervio fibular profundo; FC: músculo fibular corto; TA: músculo tibial anterior. proximal, partes proximal y distal, y, parte proximal del tercio medio, los valores promedios de distribución de los puntos y sus respectivas representaciones porcentuales en relación a la longitud de la fíbula (\%CF), juntando los lados derecho e izquierdo en ambos géneros. En la parte proximal del tercio proximal, se identificaron hasta 5 puntos de penetración (motores) P1-P5; en la parte distal de este tercio, se observaron hasta 7 puntos motores (P1-P7) y en la parte proximal del tercio medio, hasta 2 puntos motores (P1-P2) (Fig. 1).

Músculo fibular corto. Se observaron 148 puntos de penetración o motores originados desde el NFS. De éstos, 76

Tabla I. Promedios y Desviación Standard del número y distancias de los puntos de penetración o motores (P) en el músculo fibular largo, en relación al ápice de la cabeza de la fíbula y la normalización de las medidas en porcentaje de la longitud de la fíbula $(\% \mathrm{CF})$.

\begin{tabular}{|c|c|c|c|c|c|c|}
\hline \multirow[t]{2}{*}{ Tercio } & & \multicolumn{2}{|c|}{ Distancia en mm } & \multicolumn{3}{|c|}{$\% \mathrm{CF}$} \\
\hline & & $\mathbf{X}$ & DS & $\mathbf{X}$ & DS & p valor \\
\hline \multirow[t]{5}{*}{ Proximal Proximal } & $\mathrm{P} 1$ & 48,6 & 6,34 & 12,85 & 1,51 & 0,7 \\
\hline & $\mathrm{P} 2$ & 51,77 & 6,57 & 13,85 & 1,56 & 0,67 \\
\hline & P3 & 54,2 & 5,96 & 14,55 & 1,33 & 0,60 \\
\hline & $\mathrm{P} 4$ & 56,4 & 5,43 & 15,11 & 0,97 & $*$ \\
\hline & P5 & 57,89 & 6,3 & 15,6 & 1,23 & $*$ \\
\hline \multirow[t]{7}{*}{ Proximal Distal } & $\mathrm{P} 1$ & 74,02 & 11,08 & 19,83 & 2,61 & 0,11 \\
\hline & $\mathrm{P} 2$ & 86,26 & 17,85 & 23,04 & 4,11 & 0,21 \\
\hline & P3 & 92,86 & 16,93 & 24,99 & 3,94 & 0,16 \\
\hline & $\mathrm{P} 4$ & 100,96 & 16,82 & 27,06 & 3,97 & 0,95 \\
\hline & P5 & 102,92 & 13,84 & 28,16 & 3,34 & $*$ \\
\hline & P6 & 106,11 & 13,8 & 28,24 & 28,78 & $*$ \\
\hline & P7 & 108,08 & 12,79 & 2,77 & 2,67 & $*$ \\
\hline \multirow[t]{2}{*}{ Medio Proximal } & $\mathrm{P} 1$ & 131,34 & 12,63 & 34,73 & 1,86 & $*$ \\
\hline & $\mathrm{P} 2$ & 141,56 & 16,36 & 38,09 & 2,99 & $*$ \\
\hline
\end{tabular}

$\mathrm{P}<0,05 ; *$ Datos insuficientes para comparación entre promedios. 
PELOZO JR, O.; GARbELOTTI JR, S. A.; ITEZEROTI, A. M.; CRICENTI, S. V. ; DE ANGELIS, M. A. \& OLAVE, E. Estudio biométrico de la distribución de los ramos motores del nervio fibular superficial en el compartimiento lateral de la pierna en individuos Brasileiros: implicancias clínicas y variaciones. Int. J. Morphol., 36(4):1457-1452, 2018.

Tabla II. Promedios y Desviación Standard del número y distancias de los puntos de penetración o motores (P) en el músculo fibular corto, en relación al ápice de la cabeza de la fíbula y la normalización de las medidas en porcentaje de la longitud de la fíbula $(\% \mathrm{CF})$

\begin{tabular}{|c|c|c|c|c|c|c|}
\hline \multirow[t]{2}{*}{ Tercio } & & \multicolumn{2}{|c|}{ Distancia en mm } & \multicolumn{3}{|c|}{$\% \mathrm{CF}$} \\
\hline & & $\mathrm{X}$ & DS & $\mathrm{X}$ & DS & $\mathrm{p}$ valor \\
\hline \multirow[t]{3}{*}{ Medio Proximal } & P1 & 163,34 & 17,32 & 43,85 & 3,59 & 0,68 \\
\hline & $\mathrm{P} 2$ & 175,21 & 17,99 & 46,92 & 3,68 & 0,75 \\
\hline & P3 & 166,66 & 15,63 & 46,05 & 3,81 & $*$ \\
\hline \multirow[t]{2}{*}{ Medio Distal } & $\mathrm{P} 1$ & 201,42 & 18,78 & 54,22 & $*$ & 0,26 \\
\hline & $\mathrm{P} 2$ & 209,67 & 15,7 & 55,92 & $*$ & $*$ \\
\hline
\end{tabular}

$\mathrm{P}<0,05 ; *$ Datos insuficientes para comparación entre promedios.

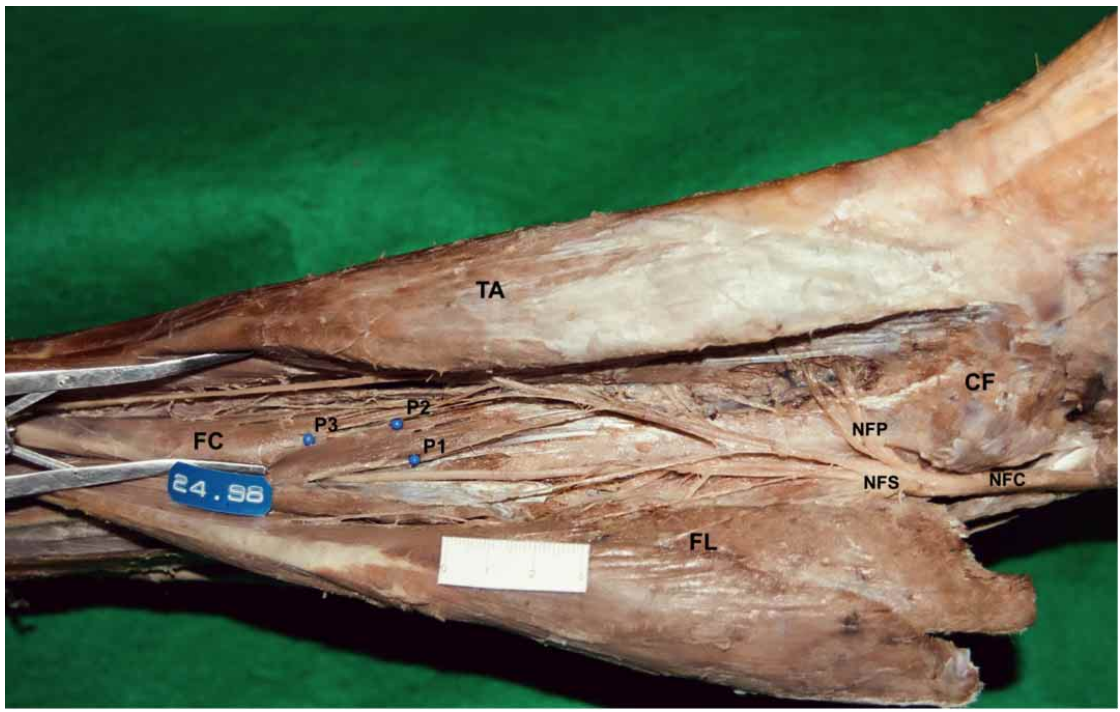

Fig. 2. Compartimiento lateral de una pierna izquierda, demostrando la ramificación del NFS en tres ramos motores (marcadores azules; P1-P3) en el tercio medio proximal para el mFC. NFC: nervio fibular común; CF: cabeza de la fíbula; NFP: nervio fibular profundo; FL: músculo fibular largo; TA: músculo tibial anterior.

\section{DISCUSIÓN}

Los clásicos textos de Anatomía así como también los escritos por cirujanos para su área, describen el NFS de manera general, señalando a éste como único nervio en la pierna, por debajo de la fascia en el compartimiento lateral (Canale, 2007). Las variaciones descritas sobre este nervio (NFS) se han relacionado principalmente con la distribución terminal de sus dos ramos hacia la piel del dorso del pie (Gabrielli et al., 2005; Barret et al.; Olave et al, 2011).

El NFS perfora generalmente la fascia profunda (crural) como una estructura única, que posteriormente se bifurca en los nervios cutáneos dorsales medial e intermedio (Gabrielli et al.; Iannoti \& Parker, 2013: Tomaszewski et al., 2017).
El estudio de las variaciones anatómicas de los nervios periféricos tiene implicaciones clínicas y quirúrgicas muy positivas, como por ejemplo, al determinar nervios para los procedimientos de transferencia nerviosa directa para restauración sensitiva-motora en lesiones complejas o en la selección de colgajos donadores potenciales para injerto autólogo, procedimiento que es difícil determinar de manera absoluta, además que las opciones posibles de donadores son limitadas y generalmente restringidas a los nervios cutáneos de los miembros (Buntic et al., 2002; Rodríguez-Lorenzo et al., 2011; Ribak et al.; Tomaszewski et al.).

Por otro lado, la presencia de variaciones puede tener consecuencias clínicas negativas como el riesgo de lesiones iatrogénicas (Prakash et al.;

Tomaszewski et al.). Según Lockhart et al. (1983), el nervio fibular común es el más lesionado del miembro inferior, ya sea directamente o por fracturas de la epífisis proximal de la fíbula, pudiendo también ser comprimido al contornear el cuello de la fíbula por un yeso mal colocado. En estos casos, el pie no puede ser evertido, además del aparecimiento de parestesia de la región sobre la fíbula y la parte medial del dorso del pie (Canale).

Por lo tanto, los estudios anatómicos utilizando cadáveres como el presentado en este artículo, continúan siendo la mejor manera de detallar los dominios anatómicos de un nervio y sus variaciones (Prakash et al.), entregando datos que puedan contribuir en el diagnóstico diferencial de una lesión.

En este sentido, el presente estudio entrega detalles sobre el número de puntos de penetración de cada nervio en 
PELOZO JR, O.; GARBELOTTI JR, S. A.; ITEZEROTI, A. M.; CRICENTI, S. V. ; DE ANGELIS, M. A. \& OLAVE, E. Estudio biométrico de la distribución de los ramos motores del nervio fibular superficial en el compartimiento lateral de la pierna en individuos Brasileiros: implicancias clínicas y variaciones. Int. J. Morphol., 36(4):1457-1452, 2018.

los músculos del compartimento lateral de la pierna y sobre las distancias de éstos respecto a un punto de referencia óseo, correlacionando estas medidas con la longitud de la fíbula.

La literatura muestra pocos estudios sobre esta temática, pudiendo mencionar entre otros, los trabajos de Bakkum et al. (1996), Reebye y Lee et al. Estos autores encontraron padrones semejantes al presente estudio con predominancia de dos o tres ramos motores para el mFC y de tres a cuatro ramos para el mFL. Aunque Olave et al. (2010) describieron tambien un predominio de de tres y cuatro ramos para este último músculo, no fue así para el mFC donde encontraron un predominio de uno a dos ramos. En otro de los estudios analizados se reportaron dos ramos para el mFL y uno para el mFC (Büyükmumcu et al.).

Es importante dejar en claro que el presente estudio trata sobre los ramos derivados del NFS, ya que el mFL también puede recibir inervación desde el NFC y en el caso del mFC, alguno de sus ramos pueden derivar del NFP accesorio (Olave et al., 2011).

Durante la disección, se observó que los ramos motores principales que se dirigían hacia los músculos, se ramificaban en su parte terminal, casi al ingresar en su vientre muscular y los ramos resultantes de esa división eran los que penetraban en el mismo. Esta característica también fue descrita por Olave et al. (2010), autores que encontraron en promedio 5 puntos motores en el mFL y 3 en el mFC.

Se determinó que la mayor frecuencia de ramos ingresando en el mFL ocurrió en la parte distal del tercio proximal, mientras que en el $\mathrm{mFC}$, se realizaron en las partes proximal y distal del tercio medio.

En el estudio realizado por Olave et al. (2011), se consideró la distancia entre el epicóndilo lateral del fémur (EPCL) y el maléolo lateral (ML), de tal modo que se dividió a ésta en tercios y asi se obtuvo que el nivel de origen de los ramos para el mFL se localizó en el tercio proximal de la distancia EPCL - ML y sus puntos motores se encontraron principalmente en este mismo tercio. En cambio, el origen de los ramos del mFC así como sus puntos motores se localizaron principalmente en el tercio medio de la distancia EPCL-ML.

En los estudios de Büyükmumcu et al. y Ribak et al. se describió que la distancia promedio desde la cabeza de la fíbula hasta la bifurcación del primer ramo motor para el $\mathrm{mFL}$ fue de $49 \mathrm{~mm}$ con distancias de 38 y $60 \mathrm{~mm}$, respectivamente, datos semejantes al obtenido en nuestra investigación, en que se registró 48,06 mm; para el mFC obtuvieron 40 y $67 \mathrm{~mm}$, respectivamente, bien distante de nuestros resultados, los cuales fueron 163,34 mm para el más proximal y 209,67 mm para el más distal.
Una forma de minimizar estas distorsiones de los valores absolutos es la normalización de las medidas en relación al tamaño de la fíbula, tal como describieron Lee et $a l$., cuyos datos indicaron que los puntos de penetración en el mFL estaban aproximadamente entre 20 y $40 \%$ del tamaño del hueso mencionado y los ramos para el mFC lo hicieron entre 40 y $60 \%$, resultados muy semejantes a los encontrados en esta investigación, donde los puntos se encontraban entre $12,85 \%$ y $38,09 \%$ en el mFL y entre $43,85 \%$ y $55,92 \%$ en el $\mathrm{mFC}$.

A pesar de las diferencias con otros estudios, mientras más detalles sobre la anatomía de la distribución nerviosa en el compartimiento lateral de la pierna, mayor será la precisión en el momento de elegir un área para colgajo de injerto autólogo y menor la ocurrencia de lesiones iatrogénicas en procedimientos quirúrgicos en la región.

PELOZO JR, O.; GARBELOTTI JR, S. A.; ITEZEROTI, A. M.; CRICENTI, S. V. ; DE ANGELIS, M.A. \& OLAVE, E. Biometric study of the distribution of the motor branches of the superficial fibular nerve in the lateral compartment of the leg in Brazilian individuals: clinical implications and variations. Int. J. Morphol., 36(4):1447-1452, 2018.

SUMMARY: The purpose of the present study was to know the distribution of the motor branches of the superficial fibular nerve (SFN) and their respective motor points in the fibular muscles in relation to the apex of the head of the fibula, dividing the lateral compartment of the leg in three regions in order to make possible a safer view of your clinical and surgical correlations. Through dissection, 60 paired legs of 30 adult cadavers, of both sexes, Brazilians, with an average age of 44.9 years, 8 being female and 22 male, were studied. After the dissection, the distances of the motor points of the NFS branches in the fibularis longus (FLm) and brevis (FBm) muscles were recorded, locating them in the proximal, middle or distal thirds. It was observed that the largest number of branches penetrated the FLm at the level of the distal part of the proximal third of the leg, while in the FBm they did so in the proximal and distal parts of the middle third of the leg. The motor branches for the FLm penetrated into the muscular belly between 48.06 and $141.56 \mathrm{~mm}$, and the branches for the FBm did between 163.34 and $209.67 \mathrm{~mm}$ of the origin of the nerve. There were no statistically significant differences between the right and left sides or between genres. Regardless of the methodological differences between the available studies, the detail of the nervous distribution in this compartment will allow a greater precision at the time of choosing an area for autologous graft flap and a lower chance of iatrogenic injuries during surgeries of the region.

KEY WORDS: Anatomy; Superficial fibular nerve; Fibular muscles; Anatomical variations. 
PELOZO JR, O.; GARbELOTTI JR, S. A.; ITEZEROTI, A. M.; CRICENTI, S. V. ; DE ANGELIS, M. A. \& OLAVE, E. Estudio biométrico de la distribución de los ramos motores del nervio fibular superficial en el compartimiento lateral de la pierna en individuos Brasileiros: implicancias clínicas y variaciones. Int. J. Morphol., 36(4):1457-1452, 2018.

\section{REFERENCIAS BIBLIOGRÁFICAS}

Agthong, S.; Huanmanop, T.; Sasivongsbhakdi, T.; Ruenkhwan, K.; Piyawacharapun, A. \& Chentanez, V. Anatomy of the superficial peroneal nerve related to the harvesting for nerve graft. Surg. Radiol. Anat., 30(2):145-8, 2008.

Bakkum, B. W.; Russell, K.; Adamcryck, T. \& Keyes, M. Gross anatomic evidence of partitioning in the human fibularis longus and brevis muscles. Clin. Anat., 9(6):381-5, 1996

Barrett, S. L.; Dellon, A. L.; Rosson, G. D. \& Walters, L. Superficial peroneal nerve (superficial fibularis nerve): the clinical implications of anatomic variability. J. Foot Ankle Surg., 45(3):174-6, 2006.

Buntic, R. F; Buncke, H. J; Kind, G. M; Chin, B. T; Ruebeck, D. \& Buncke, G. M. The harvest and clinical application of the superficial peroneal sensory nerve for grafting motor and sensory nerve defects. Plast. Reconstr. Surg., 109(1):145-51, 2002.

Büyükmumcu, M.; Ustün, M. E.; Seker, M.; Kocaog ullari, Y. \& Sag manligil, A. The possibility of deep peroneal nerve neurotisation by the superficial peroneal nerve: an anatomical approach. J. Anat., 194(Pt. 2):30912, 1999.

Canale, S. T. Cirurgia Ortopédica de Campbell. 10ª ed. São Paulo, Manole, 2007.

Ducic, I.; Dellon, A. L. \& Graw, K. S. The clinical importance of variations in the surgical anatomy of the superficial peroneal nerve in the mid-third of the lateral leg. Ann. Plast. Surg., 56(6):635-8, 2006.

Gabrielli, C.; Froehner Junior, I. \& Braga, M. T. T. Anatomical and biometric aspects of the cutaneous distribution of the superficial fibular nerve. Int. J. Morphol., 23(2): 163-70, 2005.

Iannotti, J. P. \& Parker, R. D. The Netter Collection of Medical Illustrations Musculoskeletal System. Vol. 6. Part II - Spine and Lower Limb. 2nd ed. Philadelphia, Elsevier, 2013.

Lee, J. H.; Lee, B. N.; An, X.; Chung, R. H.; Kwon, S. O. \& Han, S. H. Anatomic localization of motor entry point of superficial peroneal nerve to peroneus longus and brevis muscles. Clin. Anat., 24(2):232-6, 2011.

Lockhart, R. D.; Hamilton, G. F. \& Fyfe, F. W. Anatomia do Corpo Humano. $2^{\mathrm{a}}$ ed. Rio de Janeiro, Guanabara Koogan, 1983.

Olave, E.; Galaz, C.; Retamal, P. \& Cruzat, C. High division of superficial fibular nerve: early origin of the dorsal cutaneous nerves of the foot. Int. J. Morphol., 29(2):436-40, 2011.

Olave, E.; Galaz, C.; Retamal, P. \& Cruzat, C. Innervation of the fibularis longus and brevis muscles in Chilean individuals: Anatomic and biometric study. Int. J. Morphol., 28(4):1295-300, 2010.

Pacha, D.; Carrera, A.; Llusa, M.; Permanyer, E.; Molona, O. \& Morro, R. Clinical anatomy of the superficial peroneal nerve in the distal leg. Eur. J. Anat., 7(Suppl. 1):15-20, 2003.

Prakash; Bhardwaj, A. K.; Singh, D. K.; Rajini, T.; Jayanthi, V. \& Singh, G. Anatomic variations of superficial peroneal nerve: clinical implications of a cadaver study. Ital. J. Anat. Embryol., 115(3):223-8, 2010.

Reebye, O. Anatomical and clinical study of the common fibular nerve. Part 1: Anatomical study. Surg. Radiol. Anat., 26(5):365-70, 2004.

Ribak, S.; Fonseca, J. R.; Tietzmann, A.; Gama, S. A. \& Hirata, H. H. The anatomy and morphology of the superficial peroneal nerve. J. Reconstr. Microsurg., 32(4):271-5, 2016.

Rodríguez-Lorenzo, A.; Gago, B.; Pineda, A. F.; Bhati, M. \& Audolfsson, T. Superficial peroneal and sural nerve transfer to tibial nerve for restoration of plantar sensation after complex injuries of the tibial nerve: cadaver feasibility study. J. Plast. Reconstr. Aesthet. Surg., 64(11):1512-6, 2011.

Standring, S. Gray's Anatomy. The Anatomical Basis of Clinical Practice. 41st ed. New York, Elsevier, 2016.

Tomaszewski, K.A.; Graves, M.J.; Vikse, J.; Pe ‘kala, P.A.; Sanna, B.; Henry, B.M.; Tubbs, R.S. \& Walocha, J.A. Superficial fibular nerve variations of fascial piercing: A meta-analysis and clinical consideration. Clin. Anat. 30(1):120-5, 2017.
Dirección para Correspondencia:

Garbelotti Jr., S.

Universidade Federal de Sao Paulo

Escola Paulista de Medicina

Displina de Anatomia Descritiva e Topografica

Rua Botucatu 720, SP

BRASIL

Email: silviogarbelotti@gmail.com

Recibido : 06-07-2018

Aceptado: 26-09-2018 